\title{
Newcastle and Infectious Bronchitis Vaccine Reactions in Commercial Broilers 1
}

\author{
G. D. Butcher, DVM, Ph.D., R. D. Miles, Ph.D., and A. H. Nilipour²
}

There is considerable confusion in the commercial broiler industry relating to vaccination with Newcastle (ND) and infectious bronchitis (IB) vaccines. Questions arise over route of vaccination, age at vaccination, doses of vaccine to administer per bird, mixing of ND and IB vaccines or other vaccines, vaccine reactions, use of live vaccines versus bacterins, strains or serotypes of virus to include, need for and timing of revaccination, and others.

\section{Generic Vaccination Programs Are Costly}

Much of this confusion probably results from the attempt by integrations to use generic vaccination programs or recommendations. Each integration has its own set of variables that need to be considered in designing an effective ND and IB vaccination program. When designing a ND and IB vaccination program, for example, it is necessary to consider: 1)MG infection status, 2)Aspergillosis infection status, 3)IB and ND maternal antibody levels and uniformity of maternal titers, 4)incidence of IB and NDV field challenge, 5)virulence and serotypes of field IB and ND virus challenge, 6)status of the immune system of the birds due to infectious bursal disease or chicken infectious anemia challenge, 7)air quality in the production houses, 8)chick quality, and many other factors. On visiting integrations, it also becomes obvious that vaccination programs are often developed due to misinterpretation of vaccine responses. Thus, intensive vaccination programs may be implemented simply to attempt to limit losses resulting from adverse vaccine responses. In these cases, ND and IB field challenge are of little significance in adversely affecting production parameters and costs. Designing a vaccination program for an integration usually requires evaluation of production practices, laboratory analyses, and experimentation before the best program is identified. Thus, it is important to keep an open mind and be patient.

\section{Why Vaccinate}

Before being overwhelmed with all these questions or concerns relating to IB and ND vaccination, it is a good idea to look at the "big picture." Why are the birds being vaccinated against

1. This document is VM132, one of a series of the Veterinary Medicine-Large Animal Clinical Sciences Department, Florida Cooperative Extension Service, Institute of Food and Agricultural Sciences, University of Florida. Original publication date June 10, 2002. Visit the EDIS Web Site at http://edis.ifas.ufl.edu.

2. Gary D. Butcher, DVM, Ph.D., Diplomate, American College of Poultry Veterinarians, University of Florida College of Veterinary Medicine, Gainesville, FL., Richard D. Miles, Ph.D., Professor, Poultry Nutritionist, Department of Dairy and Poultry Sciences, Amir H. Nilipour, PhD, Director of Investigation and Quality Assurance, Grupo Melo, S.A., Panama, Republic of Panama

The Institute of Food and Agricultural Sciences is an equal opportunity/affirmative action employer authorized to provide research, educational information and other services only to individuals and institutions that function without regard to race, color, sex, age, handicap, or national origin. For information on obtaining other extension publications, contact your county Cooperative Extension Service office. Florida Cooperative Extension Service/Institute of Food and Agricultural Sciences/University of Florida/Christine Taylor Waddill, Dean. 
these diseases in the first place? Vaccination is done to provide some degree of protection against disease if the flock is infected with a field virus. Vaccination, it is hoped, will stimulate the bird's immune system so that the immune system can respond more effectively to limit losses if a challenge occurs. In unprotected birds, losses from ND and IB are more associated with decreased body weights and uniformity, poor feed conversion, and increased condemnations. Poor performance is also associated with increased medication costs, and consequently increased production costs to produce a pound of broiler meat. Rare infections with highly virulent Newcastle viruses or complicated ND and IB infections may result in greatly increased mortality.

\section{Vaccination Is not a Magic Bullet}

Vaccination against ND and IB will be of no value if the flocks are not challenged with these diseases. Vaccines do not improve feed conversion or body weights or even decrease mortality if a ND or IB field disease challenge does not occur. Actually, live vaccines do not come without costs themselves. Besides the cost of the vaccines and labor cost of administering the vaccines, vaccines will adversely affect performance. In reality, live ND and IB vaccines are mild forms of the diseases. In most cases, a vaccine virus is simply a modified or weakened field virus. Due to differences in flock health and management practices, the manner in which flocks respond can vary considerably. Proper house management that provides a stress-free environment is essential for a vaccine program to be successful. Thus, decisions on whether to vaccinate, which route of vaccination to use, which strains to use in the vaccine, or whether to revaccinate depend on the probability of field challenge. These decisions can affect production costs and performance in an integration. It is essential to have an effective vaccination program. However, improper ND and IB vaccination can be as costly to a company as the field challenge. Many general managers and field veterinarians just feel good when vaccinating the broilers, but in reality there are no guarantees that birds are protected.

\section{Normal Vaccine Reaction}

Following vaccination in poultry, the vaccine virus must infect target cells and replicate to increase their numbers to stimulate the immune system. If the vaccine is administered properly to healthy poultry, a "normal" vaccine reaction will result. Although this "normal" reaction can vary considerably, the anticipated reaction should be predictable for an integration. A good basic rule is that a mild respiratory reaction should be detected at 2 to 3 days following vaccination and should last for 5 to 7 days. This reaction should be self-limiting. If no reaction can be detected, this needs to be investigated, as likely little or no stimulation of the immune system is occurring. If the reaction is more severe than desired or is not self-limiting, this also is cause for concern and should be investigated. The key point here is to understand that a mild respiratory reaction following vaccination is normal and necessary for the vaccine to stimulate the immune system. For this normal reaction, no intervention is suggested.

\section{Why Vaccines Fail}

If the vaccine is not stored or administered properly, the virus will be inactivated and thus will not replicate and stimulate the immune system. Little or no protection will be induced. It is essential to monitor the flock following vaccination to ensure vaccination is conducted properly. When no reaction is detected, it is suggested to evaluate vaccination procedures and antibody responses. In many cases, a company may vaccinate for years and believe their vaccination program is effective, only to learn that the birds had little or no protection when field challenge does occur. A monitoring program will ensure protection is present against a potential field challenge. The fact that the birds appear healthy is not proof that the vaccine is working; it may mean they are not being challenged. Live ND and IB vaccines are fragile and must be handled according to the recommendations of the manufacturer. They should be received by the integration in good condition from the manufacturer and stored at the proper temperature in the dark (in a refrigerator). The expiration dates should be noted and recorded, and the administration procedures closely evaluated to ensure the integrity of 
the virus is maintained. Monitoring is an ongoing process.

\section{Excessive Vaccine Reactions}

Companies have complained, in many cases, of excessive reactions following ND or IB vaccination. Broilers will be vaccinated according to the integration's program and will then need to be treated with antibiotics to "buffer" the reaction by controlling secondary E. coli septicemia and airsaculitis. Many of these flocks will have poor body weights, uniformities, and feed conversions, and even experience increased mortality due to the severe and/or prolonged reactions. It is common for a company to describe a disease challenge that always starts up at 21 days of age, for example. They believe this is due to a field IB and ND field challenge. Analyses of these flocks often demonstrate that the disease challenge is really only a severe vaccine reaction following vaccination at 18 days of age. Thus, eliminating the vaccine(s) or changing the age of vaccination resolves the problem. In most of these cases, field challenge is shown to be insignificant.

The causes of excessive vaccine reactions are many. An integration manager must closely evaluate the problem and implement a plan to provide protection against field challenge, while controlling reactions to the vaccines. Some common factors associated with excessive vaccine reaction are described below. It must be determined if the losses resulting from excessive vaccine reactions are more costly than the actual field challenge to these diseases. It is not advisable to simply vaccinate with IB and NDV according to a plan recommended by an outsider. This process should be the result of laboratory study and close observation of field performance.

\section{MG infection - MG-infected flocks require} special consideration when designing the IB and ND vaccination programs. Use only those vaccines that are absolutely necessary, vaccinate at an earlier age, vaccinate using a less aggressive route, and consider antibiotic therapy to control MG during the vaccine response. MG transmission through the egg is at a low level, probably no more than $2 \%$ at peak transmission by breeders. Horizontal transmission, within a flock, will effectively permit infection of the entire flock after several weeks. Thus, earlier vaccination before infection level is increased can reduce the losses associated with vaccination. The use of antibiotics can also temporarily reduce MG infection level.

Poor uniformity of vaccine reaction - Ideally, all birds in the flock receive the same dose of vaccine at the same time. Thus, all birds respond at the same time in a predictable manner. When only a part of the flock receives the vaccine, the reaction is prolonged and is referred as a "chain" or "rolling" reaction. It is common, for example, when water-vaccinating for only a portion of the birds to consume the treated water. If this occurs, some birds receive multiple doses and others receive none. However, in the following days, vaccinated birds will horizontally shed the vaccine to pen mates. The horizontal transmission will be variable and there will be additional changes in the maternal antibody levels, further complicating the flock's reaction. The same principals apply to spray vaccination.

Selection of vaccine strains - the decision to use milder versus more aggressive vaccine strains must be carefully considered. The reaction resulting from vaccination with the $\mathrm{B} 1 \mathrm{~B} 1$ strain of ND versus the LaSota strain is considerably different. This decision must be based on the types of ND field viruses in the area and the general heath status of the flock. Use of a milder vaccine strain will not provide as much protection against a field challenge; however, the reaction will be relatively milder. Conversely, do not use a more reactive vaccine if it is not needed, as the reactions will be more severe. Infectious bronchitis vaccines selected must match those of field isolates to be effective. Laboratory studies should be completed to identify field isolates in a specific region.

Administration of vaccines - Route of application of vaccines has an effect on immune response and clinical reaction. Generally, the response and reaction to ND or IB vaccines are increasingly more pronounced following vaccine administration using fine spray, then coarse spray, eyedrop, and finally water. Companies that vaccinate by the water route and that have problems with "chain" or "rolling" reactions, should vaccinate a flock on a test farm by the eyedrop route. The eyedrop route is performed on 
a bird-by-bird basis and will allow a determination if the "rolling reaction" is caused by uneven administration or some other cause.

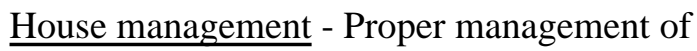
brooding temperature and litter temperature during the initial weeks is important to preventing stress, often associated with excessive vaccine reactions and respiratory diseases. Litter management and drinker sanitation to control pathogen load can prevent excessive reactions associated with secondary pathogens such as E. coli.

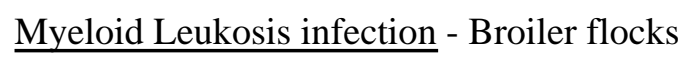
hatched from Myeloid Leukosis-infected parents often experience excessive vaccine reactions. These flocks are more prone to secondary bacterial infections associated with these reactions.

IB and ND vaccination should provide protection to a flock against field virus challenge without causing excessive reactions. An integration must determine the cost/benefit relationship of vaccinating. When problems occur, they must be recognized and efforts made to resolve the problem. Simply living with excessive reactions and the associated losses from decreased performance and increased medication costs is not an acceptable alternative. 